\title{
HYBRID APPROACH USING MOBILE SINK AND FUZZY LOGIC FOR REGION BASED CLUSTERING IN WSN
}

\author{
Amneet Kaur (1), Harpreet Kaur (2) \\ (1) Research Scholar, Department of Computer Science \& Engineering, LLRIET, Moga \\ (2) Assistant Professor, Department of Computer Science \& Engineering, LLRIET, Moga
}

\begin{abstract}
Wireless sensor network has revolutionized the way computing and software services are delivered to the clients on demand. Wireless sensor network is very important to the mankind. It consist of number of sensor called nodes and a base station. Nodes collect data and send to the base station. There are number of nodes which send data at a time. So, number of problems are occurred. So, far this nodes are divided into cluster then a cluster head will be formed. WSN is a battery powered system. When the battery is died no data send or received. So when all nodes participate for sending and receiving data then system is died earlier. Our research work proposed a new method for cluster head selection having less computational complexity. It was also found that the modified approach has improved performance to that of the other clustering approaches. The network area is divided into same sized small-small regions. Sensor nodes are randomly deployed in each predefined sub-area. Each region will have its region head $(\mathrm{RH})$ and multiple member nodes. The member nodes in a specific region will send the data to the $\mathrm{RH}$. $\mathrm{RH}$ within the region will be elected by distributed mechanism and will be based on fuzzy variables. It was found that the proposed algorithm gives a much improved network lifetime as compared to existing work. Based on our model, transmission tuning algorithm for cluster-based WSNs has been proposed to balance the load among cluster heads that fall in different regions. This algorithm is applied prior to a cluster algorithm to improve the performance of the clustering algorithm without affecting the performance of individual sensor nodes.
\end{abstract}

\section{Keywords}

Wireless, Sensor Networks, Protocol, Cluster Head, WSN, Fuzzy Logic.

\section{INTRODUCTION}

A Wireless Sensor Network or WSN is supposed to be made up of a large number of sensors and at least one base station. The sensors are autonomous small devices with several constraints like the battery power, computation capacity, communication range and memory. They also are supplied with transceivers to gather information from its environment and pass it on up to a certain base station, where the measured parameters can be stored and available for the end user. In most cases, the sensors forming these networks are deployed randomly and left unattended to and are expected to perform their mission properly and efficiently. As a result of this random deployment, the WSN has usually varying degrees of node density along its area. Sensor networks are also energy constrained since the individual sensors, which the network is formed with, are extremely energy-constrained as well. The communication devices on these sensors are small and have limited power and range. Both the probably difference of node density among some regions of the network and the energy constraint of the sensor nodes cause nodes slowly die making the network less dense. Also, it is quite common to deploy WSNs in harsh environment, what makes many sensors inoperable or faulty. For that reason, these networks need to be fault-tolerant so that the need for maintenance is minimized. Typically, the network topology is continuously and dynamically changing, and it is actually not a desired solution to replenish it by infusing new sensors instead the depleted ones. A real and appropriate solution for this problem is to implement routing protocols that perform efficiently and utilizing the less amount of energy as possible for the communication among nodes.

A wireless sensor network is a technology that emerges as a consequence of the evolution of network technology along with microelectronics and micromechanical devices. It is a new concept, a view towards the future, a clear consequence of the new steps forward in the communications field. In few words a wireless sensor network, is a network that could contain from a couple to many small nodes with sensors attached and communications capabilities to transmit and receive information. The data acquired by these sensors is then transmitted along a network from one node to another until it is collected in a central unit, normally connected to a PC. If the original expectations created around this new network concept come true, it will improve monitoring and control systems used nowadays in the environment, medical, industry, consumer, and military sectors. Due to the deployment of WSNs in unattended harsh environment conditions, it gets to be unrealistic to charge or replace their batteries. Accordingly, the energy efficient operation of wireless sensors is very important to prolong the lifetime of overall wireless sensor networks. Furthermore, in WSN, there is wireless communication among all the nodes. Moreover, the energy consumed to transmit a message is exceptionally more than the energy needed to receive the same message. Besides all this, the route of each message destined to the base station too decides the network lifetime; for example, any node with depleted battery in short routes to the base station may lead to decreased network lifetime. While, using a long route composed of many sensor nodes can significantly increase the network delay. Since many decades, a considerable measure of exploration has been performed over this subject and numerous papers have been reviewed in which various protocols have been proposed to manage and diminish the energy utilization of sensor nodes. To achieve this, the most apparent methodology is grouping sensor nodes into clusters. Clustering is one of the design methods to manage the network energy consumption efficiently, by minimizing the number of nodes that take part in long-distance communication with the base station and distributing the energy consumption evenly among the nodes in the network. In addition, to reduce energy dissipation, protocols should be robust to node failures, fault-tolerant and scalable in order to maximize system lifetime. Leach is the most widely used protocol in clustering. The theory of the algorithm is that all nodes have the equivalent initial energy, alternately elected cluster head. 


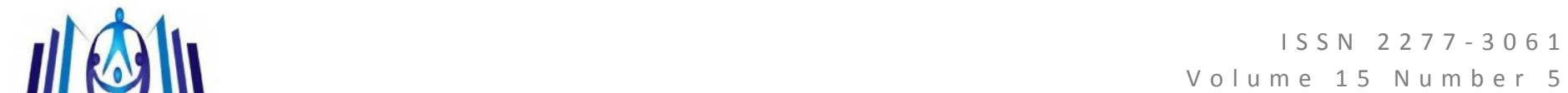

After several rounds of cycling, cluster heads consume a lot of energy, while nodes which haven't been elected as cluster head consume relatively few actually. When cluster heads transmit data to the base station, energy consumption of transmitting the same size of data packet is proportional to the distance between cluster head and the base station. The cluster heads that are far away from the base station would consume a large amount of energy while taking single-hop method to send data to the base station. After several rounds of circulation, cluster heads nearer to the base station remain with lot of energy, while cluster heads that are far away from the base station become almost blind spot detection. When more than half of nodes energy is zero, the network has lost its original meaning though remaining nodes are still working. Cluster head election is entirely based on the residual/remaining energy and distance to base station. If the $\mathrm{CH}$ will send the data directly to the BS, then there will be much loss of energy for the $\mathrm{CH}$ which is far from the $\mathrm{BS}$. If $\mathrm{CH}$ chooses the multi-hop node (relay node) for data transmission, then the node which is connected to BS will be overloaded with data of all the regions. More parameters like number of neighbors, average energy consumption should also be considered before electing the node as cluster head.

\section{LITERATURE SURVEY}

The needed detailed literature survey, to get preliminary knowledge and search scope of investigation, to implement Low energy adaptive clustering hierarchy, is explained in this section. This synopsis presents investigational studies in several energy efficient routing algorithms and its general purpose. Ding et al. (2009) presented a novel cluster-head selection algorithm. Instead of changing the threshold to 0 directly, the algorithm adjusted the threshold of each node gradually according to the roles they had played in the last round, so more nodes could have the opportunity to be CHs. Simulation results shown that the proposed algorithm outperforms LEACH in network lifetime by an average of $30 \%$ approximately. Dastgheib, Oulia \& Ghassami (2011) proposed an efficient method for clustering using fuzzy logic with appropriate inputs and combine it with the good features of LEACH. This method was fully distributed. Therefore its speed was more and its energy consumption was less than centralized methods. Also proposed method will resolve the weaknesses of LEACH and more efficient than the existing methods. Sharma \& Kumar (2012) proposed F-MCHEL, a homogeneous energy protocol. In LEACH protocol the clusters were formed randomly on the basis of threshold values; whereas, in the proposed protocol a fuzzy logic approach was used to elect the cluster-head based on two descriptors - energy and proximity distance .Out of these elected cluster heads one Master cluster head had been elected.The cluster head which had the maximum residual energy was elected as Master cluster head. In conventional Leach approach all the Cluster heads were used to send the aggregated information to the base station, however in the proposed protocol only Master cluster head sends the aggregated information to the base station. Simulation results on MATLAB shows that the proposed protocol provides higher energy efficiency, better stability period and lower instability period as compared to LEACH protocol in spite of overhead of election of Master cluster head. Results obtained shown that an appropriate Master cluster-head election can drastically reduce the energy consumption and enhance the lifetime of the network. Beiranvand, Patooghy, Fazeli (2013) proposed an energy efficient routing algorithm which saved a significant portion of inner-network communications energy. The proposed routing algorithm selects sensor nodes with higher residual energy, more neighbors, and lower distance from the Base Station (BS) as Cluster Head (CH) nodes. Then, it managed sensor nodes appropriately and constructs clusters such a way to maximize WSN lifetime and minimize average energy dissipation per each sensor node. To evaluate the proposed routing algorithm, various simulations had been carried out by using of MATLAB simulator. The proposed routing algorithm was compared to the previous proposed algorithms e.g., LEACH, DBS, and LEACH-C algorithms. Results of the simulations show that the proposed routing algorithm improved the WSN performance at least $65 \%$, reduces the energy consumption of the WSN up to $62 \%$, and improves the successfully delivered packet ratio by at least $56 \%$ as compared to the previous routing algorithms. Nitesh, Azaruddin, Jana (2015) proposed a distributed algorithm to design an energy efficient cluster base WSN. Also proposed a local recovery mechanism for the orphan sensor nodes, which were generated due to the failure of any cluster head. The proposed algorithm was based on cost function which was formulated with several parameters such as residual energy, distance and cluster cardinality. The algorithm was simulated rigorously over several performance metrics and the results acquired were compared with some existing algorithms to demonstrate its effectiveness. Tang \& Chaw (2015) described the Wireless Sensors Networks (WSN) fault diagnosis problem as a pattern classification problem and introduced a newly developed algorithm, Neighborhood Hidden Conditional Random Field (NHCRF), for determining hidden states between sensors. The health conditions of WSN were determined by using the NHCRF model to estimate the posterior probability of different faulty scenarios. The NHCRF model could improved the WSN fault diagnosis because it had relaxed the independence assumption of the Hidden-Markov model. To enhance the robustness and anti-noise ability of the NHCRF, the concept of nearest neighbors was used when estimating dependencies. In the paper, a 200-sensor-node WSN was used to show that the proposed NHCRF method can deliver excellent and effective results for WSN-health diagnosis. The study also presented thorough comparative results that indicate that method can deliver superior classification performance compared with other methods. Alduais et al. (2016) proposed a method to reduce the number of data transmissions as well as decrease the amount of data that will result in prolonging the lifetime of the network. The proposed method aimed to reduce the number of transmitted messages via a node supporting single and multiple sensors based on the relative change or relative differences between the current and last sensor measured values transmitted. The results shown the proposed method appeared to deliver the best performance in terms of reducing the number of messages transitions and packets size, in the study the mean percentage of reducing the number of messages transmissions was $74 \%$, and $80 \%$ was the mean percentage reduce of node packet size.From the results, it's clear that reduced number of packet's transitions and reduced node's packet size, will result in a decreased energy consumption as well as prolonging the lifetime of the system.

\section{RESEARCH MOTIVATION}




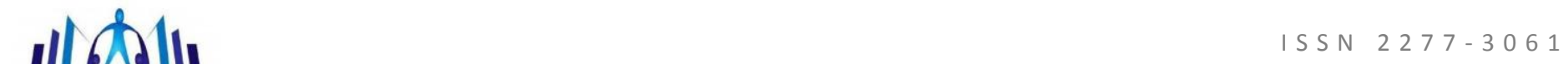

Due to the deployment of WSNs in unattended harsh environment conditions, it gets to be unrealistic to charge or replace their batteries. Accordingly, the energy efficient operation of wireless sensors is very important to prolong the lifetime of overall wireless sensor networks. Furthermore, in WSN, there is wireless communication among all the nodes. Moreover, the energy consumed to transmit a message is exceptionally more than the energy needed to receive the same message. Besides all this, the route of each message destined to the base station too decides the network lifetime; for example, any node with depleted battery in short routes to the base station may lead to decreased network lifetime. While, using a long route composed of many sensor nodes can significantly increase the network delay.

- Cluster head election is entirely based on the residual/remaining energy and distance to base station.

- If the $\mathrm{CH}$ will send the data directly to the $\mathrm{BS}$, then there will be much loss of energy for the $\mathrm{CH}$ which is far from the BS.

- If $\mathrm{CH}$ chooses the multi-hop node (relay node) for data transmission, then the node which is connected to BS will be overloaded with data of all the regions.

- More parameters like number of neighbors, average energy consumption should also be considered before electing the node as cluster head.

\section{RESEARCH OBJECTIVES}

- To review the existing energy efficient algorithms in wireless sensor networks.

- To propose an enhanced energy saving algorithm with base station mobility \& categorize the Cluster heads as boundary $\mathrm{CH}$ and non-boundary $\mathrm{CH}$.

- To replace the faulty cluster head with the newly elected cluster head mechanism process based on residual energy, number of neighbors and average energy consumption.

- $\quad$ To compare the results of the proposed work with the existing work using throughput, average energy consumption \& network delay metrics.

\section{RESEARCH METHODOLOGY}

- The various steps that are followed are as follows:

- The network area is of $n \times n$ meters.

- Divide this network area into same sized small-small regions (in meters each).

- All nodes will sends their energy level and location information to the sink.

- Sensor nodes are randomly deployed in each predefined sub-area. Each region will have its region head $(\mathrm{RH})$ and multiple member nodes. The member nodes in a specific region will send the data to the $\mathrm{RH}$.

- $\mathrm{RH}$ within the region will be elected by distributed mechanism and will be based on fuzzy variables like highest residual energy, more number of neighbors and average energy consumption.

- $\quad$ Each $\mathrm{RH}$ will create a TDMA schedule for the member nodes to transmit the data.

- Base station is movable along the boundary.

- $\quad$ BS is not energy constrained. It means it is having sufficient amount of energy for retrieving the data from the region heads and movement along the boundary.

- The Region heads are divided into 2 classes:

- $\quad$ Boundary $\mathrm{RH}$

- Non-Boundary RH

- Only boundary $\mathrm{RH}$ will send the data to the BS only when it will come within its region.

- Boundary RH will have sufficient amount of time to send the data to the base station when BS is within its boundary.

- When boundary $\mathrm{RH}$ is sending its data to the $\mathrm{BS}$, the other nodes will not send the data to that specific boundary $\mathrm{RH}$.

- Non-boundary region heads will evaluate the lower bound limit and upper bound limit of both x-axis and y-axis to evaluate the next relay node. It will choose the relay node on the path where BS is closely located which will reduce the transmission delay and will also reduce the data packet size. 


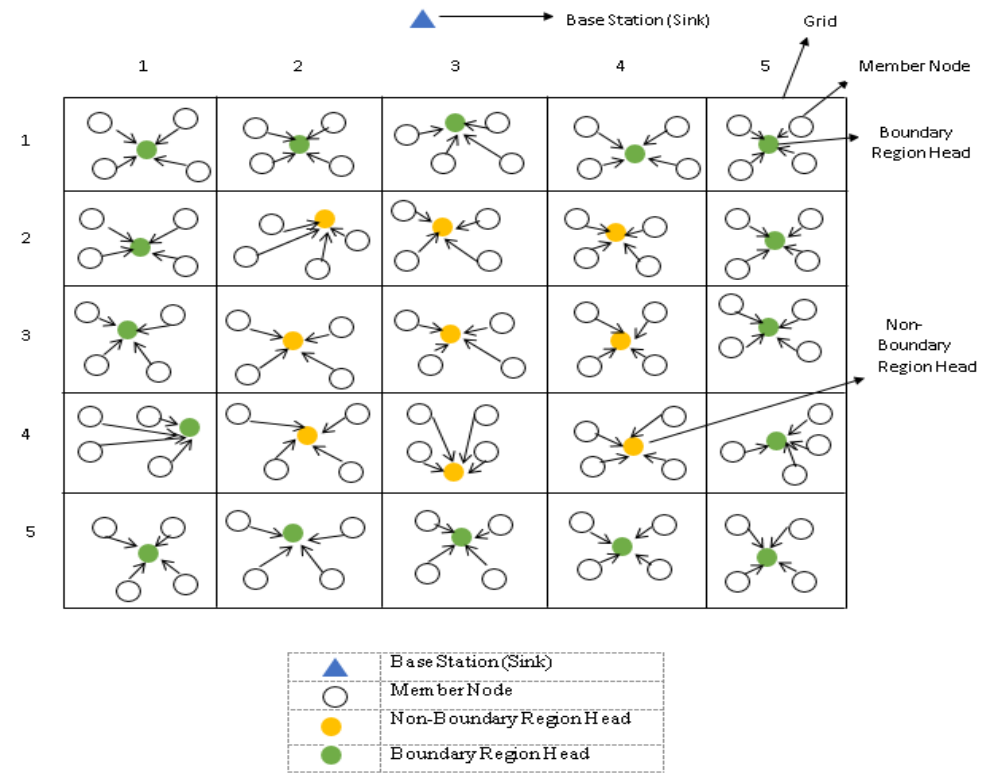

Figure 1. Region Based Clustering

The following figure 1 represents the region based clustering. The whole network is divided into small-small regions. The sensor nodes are randomly deployed in these sub-areas. Each region has its region head. The regions along the boundary have boundary region head and the regions that are not along the boundary have the non-boundary region heads. The member nodes send their data to the region heads.

\section{FUZZY RULE BASE SYSTEM}

Multiple parameters have been used for the election of region head. The Distance to base station, number of neighbors and residual energy are evaluated for each and every node in the region for the election mechanism. The decision of selection of region head is based on different scenarios. All the possible cases are listed in the table 1.

Table 1. Fuzzy Rule Base System

\begin{tabular}{|c|c|c|c|c|}
\hline S.No & $\begin{array}{c}\text { Distance of } \\
\text { Base Station }\end{array}$ & $\begin{array}{c}\text { Residual } \\
\text { Energy }\end{array}$ & $\begin{array}{c}\text { Number of } \\
\text { Neighbors }\end{array}$ & $\begin{array}{c}\text { Chance to } \\
\text { select Region } \\
\text { Head }\end{array}$ \\
\hline 1 & Far & Low & High & Less \\
\hline 2 & Far & High & Low & Less \\
\hline 3 & Far & High & High & Medium \\
\hline 4 & Close & Low & Low & Less \\
\hline 5 & Close & Low & High & Medium \\
\hline 6 & Close & High & Low & Medium \\
\hline 7 & Close & High & High & High \\
\hline
\end{tabular}




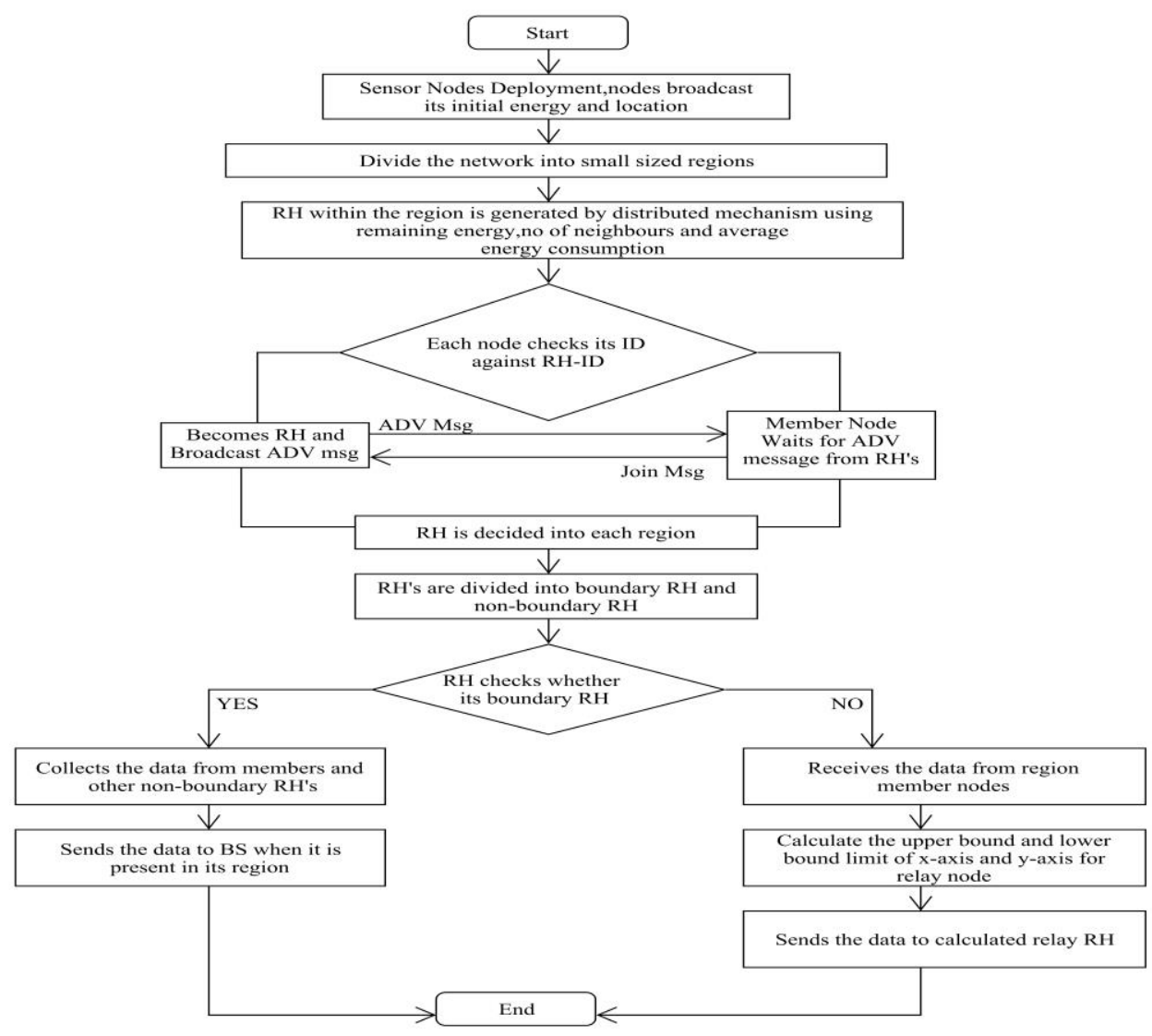

Figure 2. Flow of work

\section{RESULTS AND DISCUSSION}

This section presents the simulation results of the work done and the proposed approach. The proposed approach has been implemented in MATLAB. The parameters required for the simulation is shown in the following Table 4.1.

Table 2. Simulation Parameters

\begin{tabular}{|c|c|}
\hline Network Parameters & Values \\
\hline Network Size & $100 \mathrm{~m}^{*} 100 \mathrm{~m}$ \\
\hline Number of Sensor Nodes & 200 \\
\hline Initial Energy of Sensor Nodes & $0.5 \mathrm{~J}$ \\
\hline Packet Size & $4000 \mathrm{bits}$ \\
\hline Data Aggregation Energy consumption & $5 \mathrm{~nJ} / \mathrm{bit}$ \\
\hline Amplification Energy (Cluster to BS) $\mathrm{d}>=\mathrm{d} 0$ & Efs $=10 \mathrm{pJ} / \mathrm{bit} / \mathrm{m} 2$ \\
\hline Amplification Energy (Cluster to BS) $\mathrm{d}<=\mathrm{do}$ & $\mathrm{Emp}=0.0013 \mathrm{pJ} / \mathrm{bit} / \mathrm{m} 2$ \\
\hline
\end{tabular}

The network area is of $100^{\star} 100 \mathrm{~m}$. The network area is divided into small regions of $20 * 20 \mathrm{~m}$ each. Each region has its own cluster head. The base station is movable. It will move along the boundary of the network. The region heads are divided into two classes namely Boundary Region head and Non-Boundary region heads. Boundary $\mathrm{RH}$ will send the data to the BS only when it will come within its region. When boundary $\mathrm{RH}$ is sending its data to the BS, the other nodes will not send the data to that specific boundary $\mathrm{RH}$ and Non-boundary region heads will evaluate the lower bound limit and upper bound limit of both $x$-axis and $y$-axis to evaluate the next relay node. It will choose the relay node on the path where BS is closely located. 


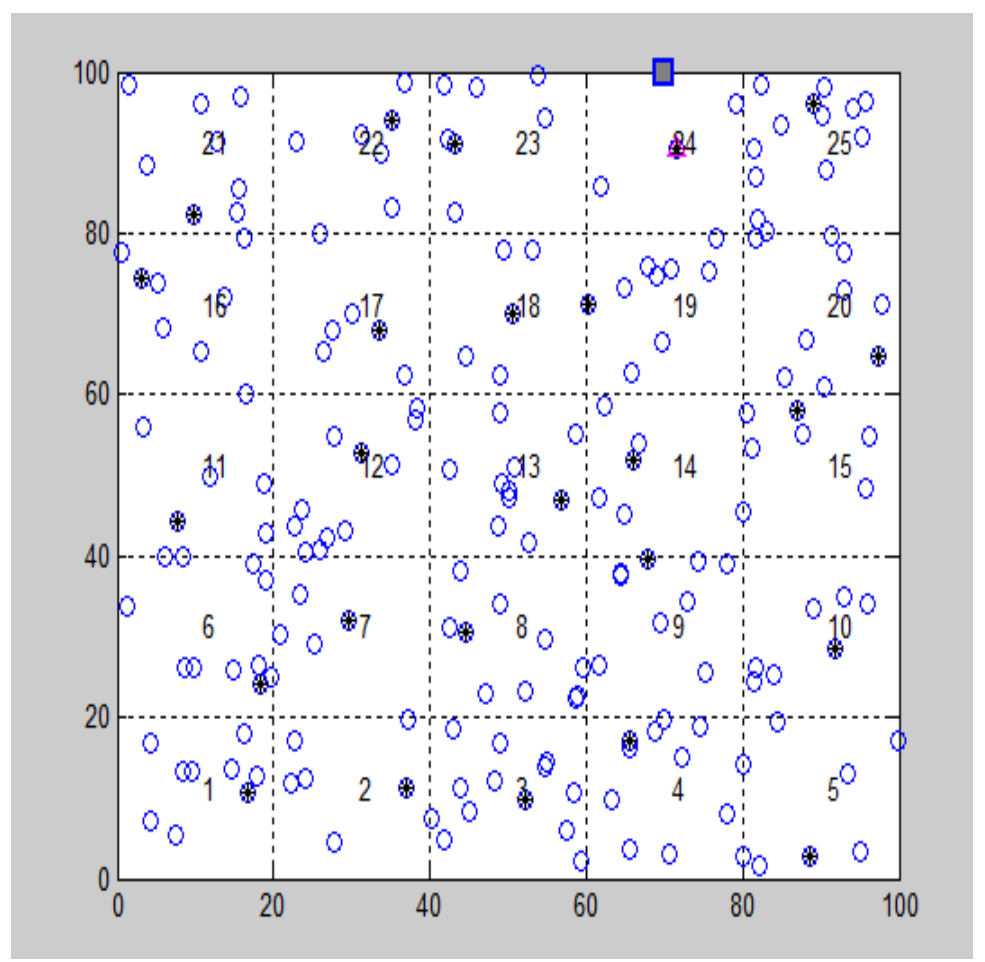

Figure 3. Network Area

The following figure represents the network where all the nodes are alive. The figure also represents the sink node as well as source nodes.

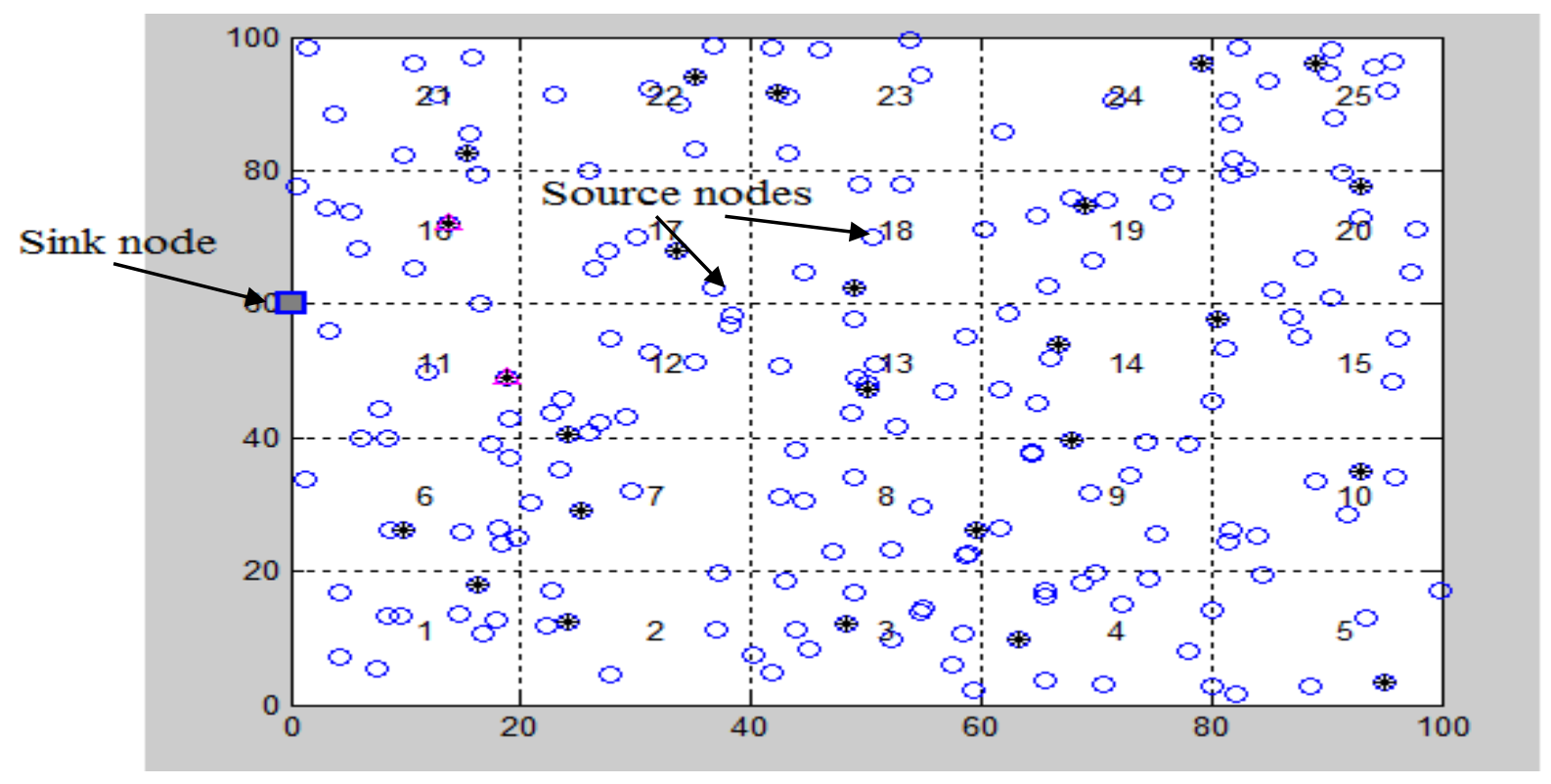

Figure 4. Network when nodes are Alive

The following figure represents the network where some of the nodes are dead. With the passage of the time the energy of the nodes decreases. So the nodes become dead.

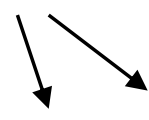




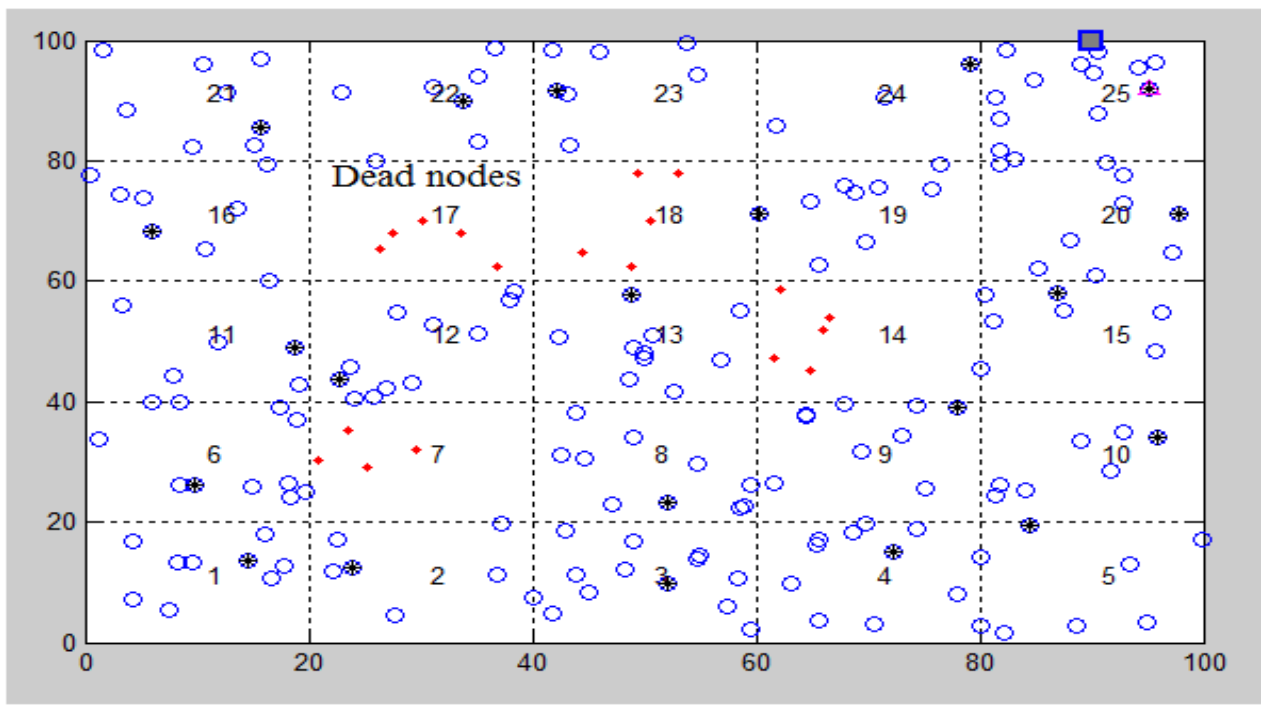

Figure 5. Network when some nodes are Dead

\section{NETWORK LIFE TIME}

The lifetime of network depends upon the number of Dead \& Alive Nodes. The proposed scheme plays an important role to achieve better network life time. Figure 5\& Figure 6 clearly represents the longer stability of the proposed scheme. An equal energy is consumed by each node in each round and some nodes get dead with variation of time. The proposed scheme achieves more stability period and longer network lifetime.

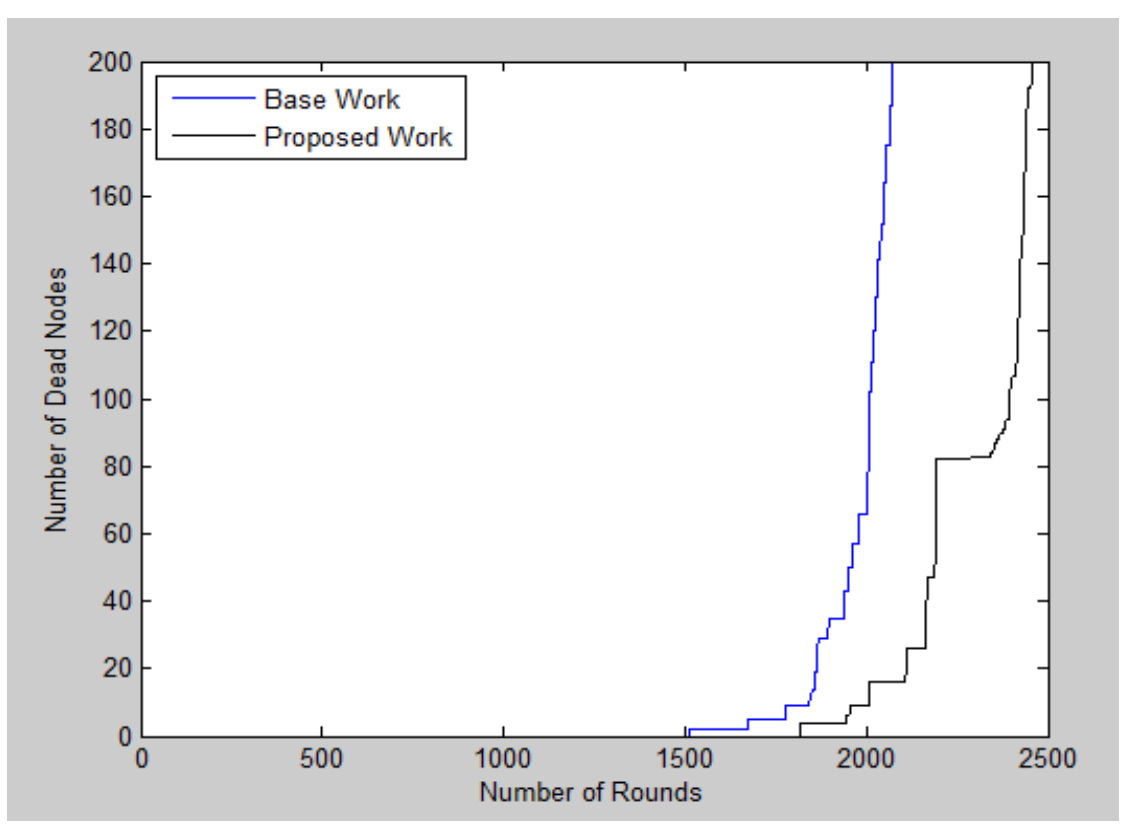

Figure 5. Analysis of dead nodes

\section{COMPARISON BETWEEN EXISTING WORK \& PROPOSED WORK}

Table 3. Comparison

\begin{tabular}{|c|c|c|c|c|c|c|}
\hline $\begin{array}{c}\text { ENERGY } \\
\text {.50J }\end{array}$ & \multicolumn{3}{|c|}{ EXISTING WORK } & \multicolumn{3}{c|}{ CURRENT WORK } \\
\hline S.No. & FND & MND & LND & FND & MND & LND \\
\hline 1 & 1367 & 2175 & 2249 & 1696 & 2408 & 2455 \\
\hline 2 & 1512 & 2010 & 2070 & 1818 & 2393 & 2456 \\
\hline
\end{tabular}




\begin{tabular}{|c|c|c|c|c|c|c|}
\hline 3 & 1645 & 2009 & 2068 & 1811 & 2402 & 2452 \\
\hline 4 & 1740 & 1993 & 2066 & 1907 & 2402 & 2455 \\
\hline 5 & 1796 & 1974 & 2074 & 1899 & 2409 & 2452 \\
\hline 6 & 1665 & 1991 & 2087 & 1689 & 2398 & 2461 \\
\hline 7 & 1709 & 2017 & 2058 & 1854 & 2409 & 2455 \\
\hline 8 & 1496 & 1992 & 2087 & 1735 & 2419 & 2461 \\
\hline 9 & 1794 & 1999 & 2092 & 1883 & 2398 & 2458 \\
\hline 10 & 1649 & 2008 & 2085 & 1863 & 2407 & 2450 \\
\hline
\end{tabular}

From the above Table 3 ,where various experiments has been done shows that the proposed work is more better than the existing work. It also shows that proposed model has high network lifetime efficiency as compared to the existing model.

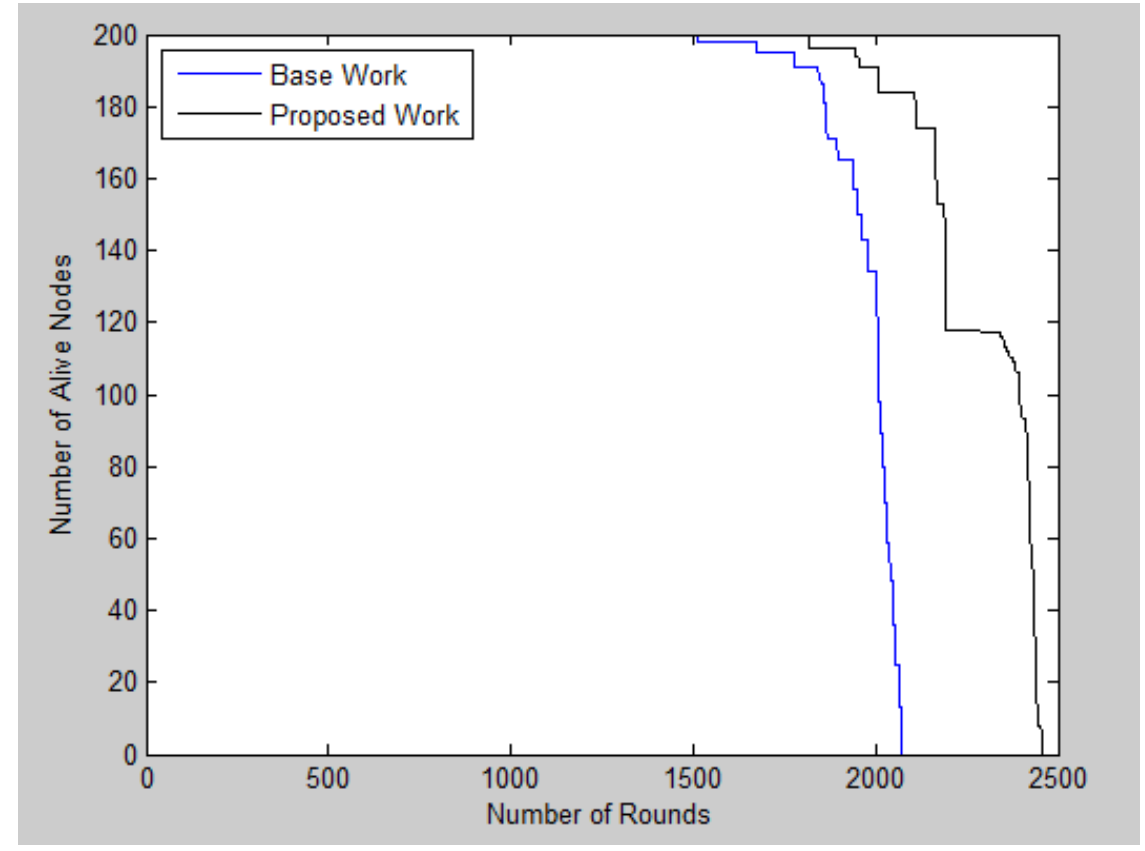

Figure 6. Analysis of Alive nodes

We can see that almost all the sensors are alive for the proposed algorithm for quite long period of time, hence extending the network efficiency. For existing algorithm, as no. of dead sensors will increase, their efficiency will decrease.

\section{THROUGHPUT}

The throughput is the fundamental parameter which checks the efficiency of the network in terms of data transfer. It is calculated as the successful number of message delivered over a communication channel and is measured in bits per second(bps).It represents the number of packets received by each node in the network over the time interval taken due packets delivery. Throughput is measured as:

\section{Throughput $=\sum \underline{\text { Number of all delivered packets }}$ \\ Receiving time interval length}

As illustrated in Figure 7, the throughput comparison of existing and proposed algorithm. Throughput is also defined as the packets that successfully reached at the sink which means that the data received at sink is maximum with less packet dropping. The proposed algorithm achieves high throughput than existing algorithm as shown. Number of packets received by sink depends upon the number of Alive nodes present in the network. More the Alive nodes, large number of packets are received by the destination sink node which improves the overall throughput of the network. 


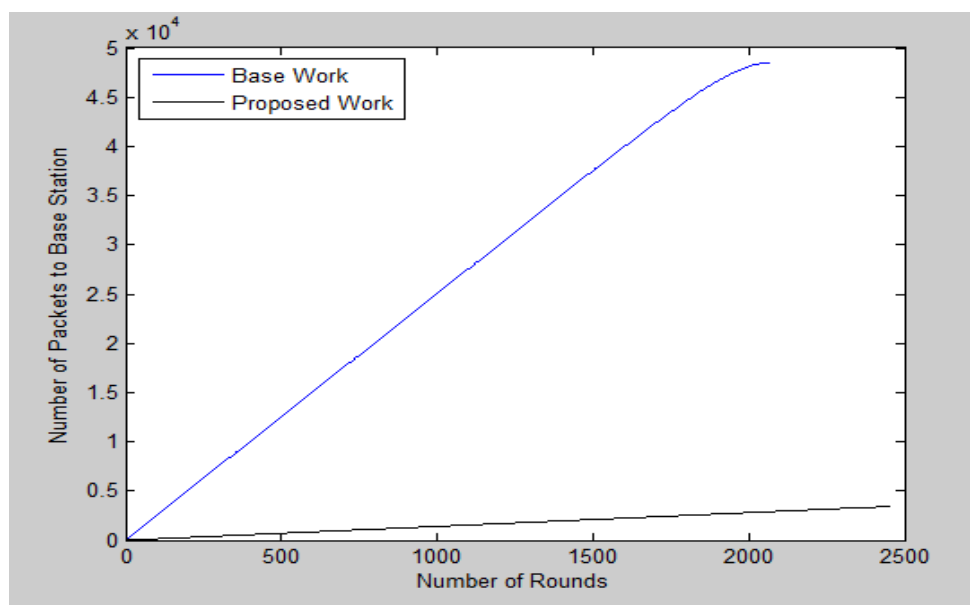

Figure 7. Throughput of the network

\section{ENERGY CONSERVATION}

Figure 8 represents the average energy consumed by the network during each round of the transmission time. The average energy consumed by the proposed algorithm is minimum as compared to the existing algorithm.

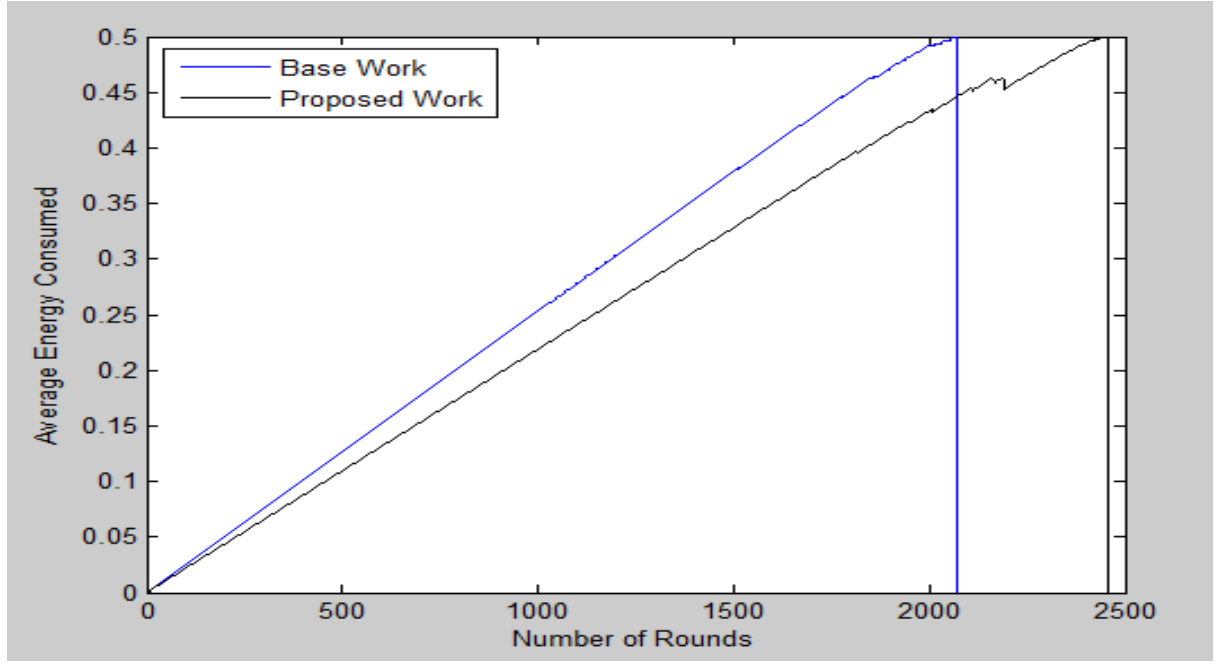

Figure 8. Energy consumed by the nodes

\section{AVERAGE REMAINING ENERGY}

In wireless networking, average remaining energy means the remaining lifetime of a wireless link on arrival of a new packet.

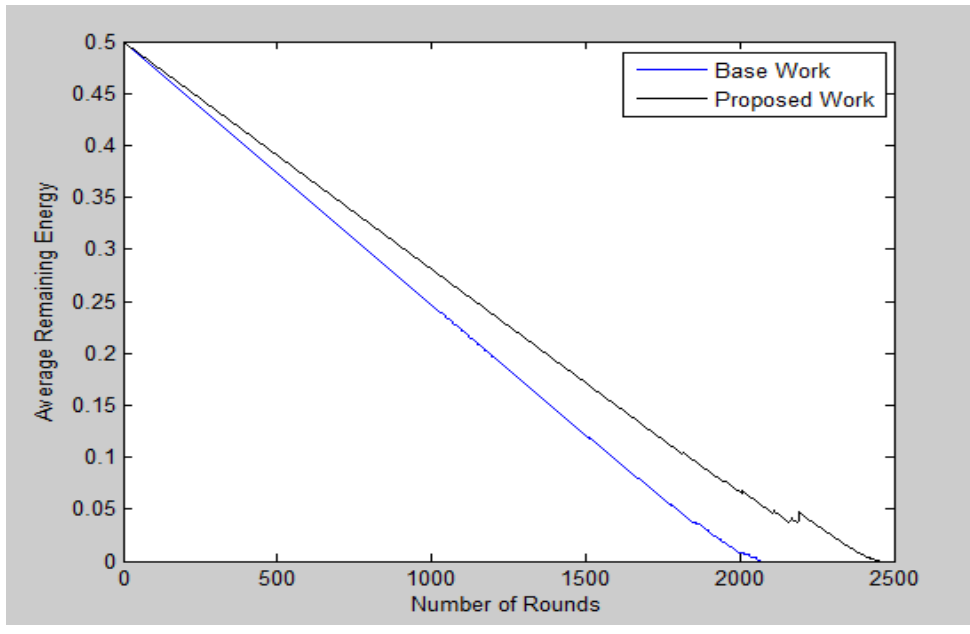

Figure 9. Average Remaining Energy 


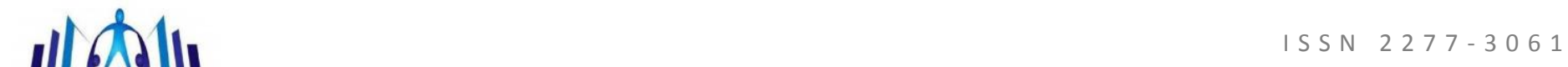

\section{NETWORK DELAY}

The number of packets sent to the cluster head by the sensor nodes are available inside the figure 10. This figure illustrates the packets sent to the cluster head in the existing work and the present work. The present work is sending more number of packets to cluster head in comparison with the existing work as the network lifetime of the present work is comparatively more than the network lifetime of the existing work. The packets sent to the cluster head are shown for different number of rounds.

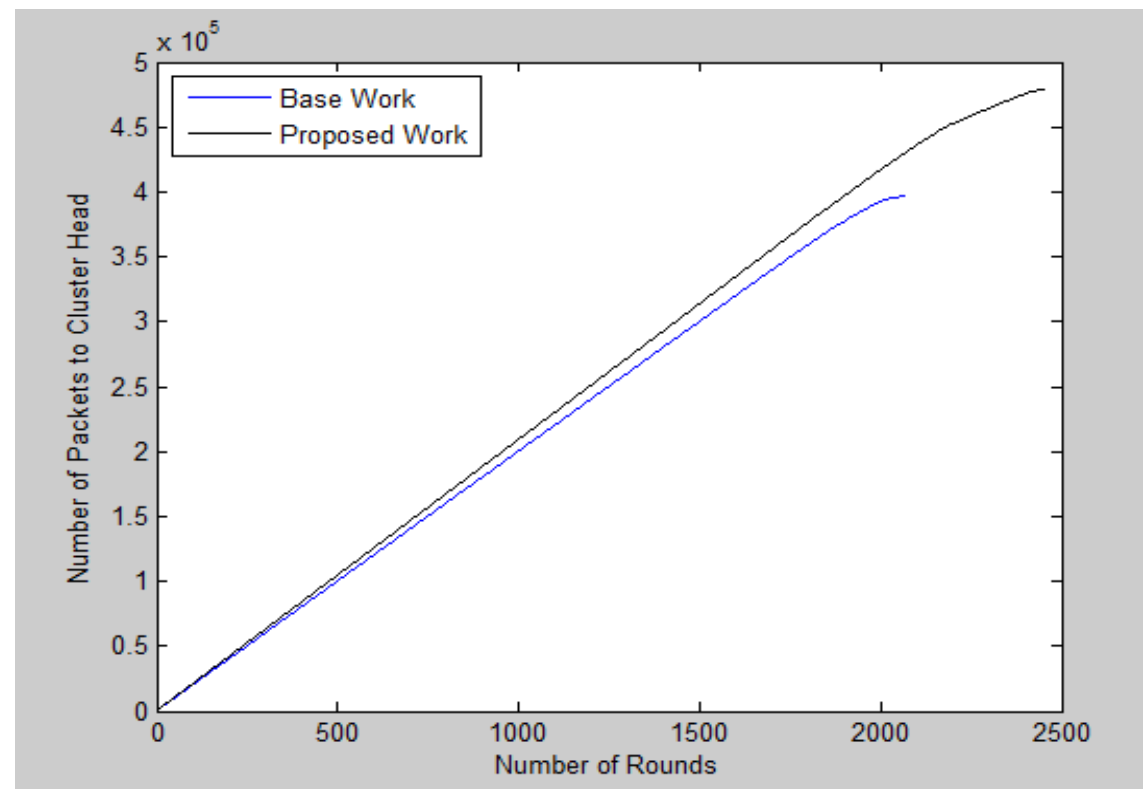

Figure 10. Network delay

\section{CONCLUSION AND FUTURE SCOPE}

The lower cost and easier installation of the WSNs than the wired counterpart pushes industry and academia to pay more attention to this promising technology. Large scale networks of small energy-constrained sensor nodes require techniques and protocols which are scalable, robust, and energy-efficient. Furthermore, data aggregation techniques are only performed by $\mathrm{CHs}$. Our thesis work included the region based clustering, cluster head selection and energy efficient communication using mobile base station. Since it was earlier proposed that clustering improves the network lifetime. We modified the region based clustering by dividing the network area into $\mathrm{n}$ regions with cluster head chosen for each region and proposed a new method for cluster head selection having less computational complexity. It was also found that the modified approach has improved performance to that of the other clustering approaches. We have used the mobile base station with controlled trajectory path as a reference to compare the performance of each of the clustering methods. It was found that the proposed algorithm gives a much improved network lifetime as compared to existing work. In this thesis, the nodes are divided into multiple regions depending upon the network area. The region height and width can be controlled according to the requirement of the network. We virtually divide the network into regions according to the proximity to the BS denoting the closest region as the boundary zone and the farthest region as the non-boundary zone. The nodes have been classified according to the region that they fall in. Based on our model, transmission of packets for cluster-based WSNs has been proposed to balance the load among cluster heads that fall in different regions. This algorithm is applied prior to a cluster algorithm to improve the performance of the clustering algorithm without affection the performance of individual sensor nodes. As a result, the network lifetime has been prolonged. By analyzing the results and graphs, we have reached up to the solution that by changing the clustering and communication mechanism, the overall lifetime of the complete network can be improved. In all of the methods discussed above the energy parameter is taken into consideration only during cluster head selection. The performance may be increased by considering energy as a parameter during clustering itself. Multiple number of experiments have been conducted to evaluate the effectiveness of the experiments. Due to the potential deployment in uncontrolled and harsh environments and due to the complex architecture, wireless sensor networks are and will be prone to a variety of malfunctioning. In future work, the detection accuracy for the nodes in the Wireless Sensor Network can be calculated where detection accuracy depicts the ratio of the number of faulty sensors detected to the total number of faulty sensors in the network. In the present work, we have improved the overall lifetime of the network but the faults occur inside the WSN can be analyzed in the future in real time scenarios.

\section{REFERENCES}

[1] Alduais, N. A. M., Abdullah, J., Jamil, A. and Audah, L., "An Efficient Data Collection and Dissemination for IOT based WSN," IEEE, pp.1-6, 2016.

[2] Abusaimeh, H., and Yang, S.H., "Dynamic cluster head for lifetime efficiency in WSN," International Journal of Automation and Computering, pp. 48-54, 2009. 


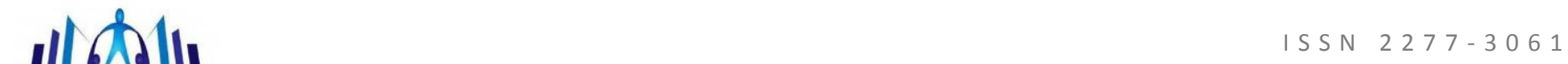

[3] Ali, A.W., and Permanand, "Energy Efficiency in routing protocol and data collection approaches for WSN : A Survey", International Conference on Computing, Communication and Automation, pp.540-545, 2015

[4] Ali, Q., AbdulMaowjod, A. and Mohammed, H., "Simulation and Performance Study of Wireless Sensor Network (WSN) Using MATLAB," Iraq J. Electrical and Electronic Engineering, vol. 7, pp. 112 - 119, 2011.

[5] Beiranvand, Z., Patooghy, A. and Fazeli, M., "I-LEACH:An Efficient Routing Algorithm to Improve Performance \& to Reduce Energy Consumption in Wireless sensor Networks", Conference on Information and Knowledge Technology, pp.13-18, 2013.

[6] Bendigeri, K.Y. and Mallapur, J.D., "Analysis of Fault Strategies in Wireless Sensor Networks", National Conference on Recent advances in Electronics \& Computer Engineering, pp.46-51, 2015.

[7] Bengherbia, B., Chadli, S., Zmirli, M.O. and Toubal A., "A MicroBlaze Based WSN Sink Node Using XBee Transceiver," IEEE, pp. 831-834, 2016.

[8] Bera, S., Misra, S., Roy, S.K. and Obaidat,M.S., "Soft-WSN: Software-Defined WSN Management System for loT Applications," IEEE, pp. 1-8, 2016.

[9] D. Peng, Q. Zhang, "An energy efficient cluster-routing protocol for wireless sensor networks", IEEE International Conference on Computer Design and Applications (ICCDA), Vol. 2, pp. 530-533, 2010.

[10] Dastagheib, J., Oulia and Ghassami, M.R.S., "An Efficient Approach for clustering in wireless sensor network using fuzzy logic", International Conference on Computer Science and Network Technology (ICCSNT), vol.3, pp.1481-1486, 2011.

[11] Dietrich, I. and Dressler, F., "On the Lifetime of Wireless Sensor Network," ACM Transaction on Sensor Network, vol. 5, pp. 1 - 38, 2009.

[12] Ding, R., Yang, B., Yang, L. and Wang, J., "Soft Threshold Based Cluster-head Selection Algorithm for Wireless Sensor Networks", International Conference on Sensor Technologies and Applications, pp.526-530, 2009.

[13] Gajjar, S., Choksi, N., Sarkar, M. and Dasgupta, K., "Comparative analysis of Wireless Sensor Network motes", Proc. of International Conference on Signal Processing and Integrated Networks, pp. 426 - 431, 2014.

[14] Gajjar, S., Sarkar, M. and Dasgupta, K., "Cluster Head Selection Protocol using Fuzzy Logic for Wireless Sensor Networks", International Journal of Computer Applications, vol.97, pp.38-43, 2014.

[15] Gupta, A.L. and Shekokar, N., "A Novel Approach to Improve Network Lifetime in WSN by Energy Efficient Packet Optimization," IEEE, pp. 117-122, 2016.

[16] Harsha, P.M. and Kanakaraju, R., "Network Lifetime Enhancement of Clustering Approach Using Handoff Mechanism in WSN", IEEE, pp.809-812, 2016.

[17] Hongjuan, Li., Kai, Lin. and Kequi, Li., "Energy-efficient and high-accuracy secure data aggregation in wireless sensor networks", Computer Communication, pp.591-597, 2011.

[18] Hou K.M., Vaulx C.D., Shi H., "Development of a Resource-efficient and Fault tolerant Wireless Sensor Network System", IEEE, pp.122-127, 2015.

[19] K, R. and Neduncheliyan, DR.S., "Cluster Based fault Tolerance Using Genetic Algorithm in Wireless Sensor Network", International Conference on Information Communication And Embedded System, pp.1-4, 2016.

[20] Kaschel, H. and Ortega, O., "Energy efficiency in routing protocols applied to WSN," IEEE,pp.1-8, 2016.

[21] Khudonogova, L.I. and Muravyov, S.V., "Feasibility estimation of creating fault-tolerant prioritized transmission scheme in WSN", IEEE, pp.1-4, 2015.

[22] Kumari, R. and Nand, P., "Performance Comparison of various Routing Protocols in WSN and WBAN," IEEE, pp. 427-431, 2016.

[23] Kushal, B.Y. and Chitra, M., "Cluster Based Routing Protocol to Prolong Network Lifetime through Mobile Sink in WSN", IEEE, pp.1287-1291, 2016.

[24] Madhupatil and Sharma, C., "Energy Efficient Cluster Head Selection to Enhance Network Connectivity for Wireless Sensor Network",IEEE,pp.175-179, 2016.

[25] Maraiya, K., Kant, K. and Gupta, N., "Wireless sensor network: a review on data aggregation". International Journal of Scientific \& Engineering Research, vol. 2, pp. 1-6, 2011.

[26] Matsui,T. and Nishi,H., "Analysis and Implementation of WSN with Route Selection Considering Energy Consumption",IEEE, pp.1-7, 2016.

[27] Min, H.Y. and Zaw, W., "Energy Efficient,Fault Tolerant Routing LEACH (EF-LEACH) Protocol for Wireless Sensor Networks", International Conference on Advances in Engineering and Technology, pp.36-40, 2014.

[28] Mohan, B.A. and Sarojadev, H., "A Hybrid Approach for Data collection using Multiple Mobile Nodes in WSN (HADMMN)", IEEE, pp.711-714, 2016 
[29] Natarajan, H. and SudhaSelvaraj, "A Fuzzy Based Predictive Cluster Head Selection Scheme for Wireless Sensor Networks", International Conference on Sensing Technology, pp.560-566, 2014.

[30] Nitesh, K., Azharuddin, Md. and Jana, P.K.,"Energy Efficient Fault -Tolerant Clustering Algorithm for Wireless Sensor Networks", IEEE, pp.234-239, 2015.

[31] Pavithra, H., Shivashankar and Poornima, G.R., "An Efficient Mobile Sink path Selection approach for WSN's”,IEEE, pp.151-155, 2016

[32] Ran, G., Zhang, H. and Gong, S.H., "Improving on LEACH Protocol of Wireless Sensor Networks Using Fuzzy Logic", Journal of Information \& Computational Science, pp.767-775, 2010.

[33] Shahabuddin, M.D., Hasbullah, H. and Aziz, I.A., "Eliminary Framework of Topology Control Algorithm Ahieve Node's Energy Efficiency," IEEE, pp. 259-263, 2016.

[34] Sharma, T. and Kumar, B., "F-MCHEL: Fuzzy Based Master Cluster Head Election Leach Protocol in Wireless Sensor Network", International Conference Journal of Computer Science and telecommunications, vol.3, Issue 10, pp.8-13, 2012.

[35] Shukla, K.V. and Bahi,C.M., "Energy efficient routing protocol LEACH for wireless sensor network", IEEE Communications Surveys, vol.15, pp. 551-591, 2013.

[36] Singh, O., Rishiwal, V. and Yadav, M., "Energy Trends of Routing Protocols for H-WSN," IEEE, pp.1-4, 2016

[37] Srivastava, J.R. and Sudarshan, T.S.B., "ZEEP: Zone based Energy Efficient Routing Protocol for Mobile Sensor Networks", in the Proceedings of the International Conference on Advances in Computing,Communications and Informatics(ICACCI), pp. 990-996, 2013.

[38] Tang, P. and Chow, T.W.S., "Wireless Sensor Networks Conditions Monitoring and Fault Diagnosis Using Neighborhood Hidden Conditional Random Field", IEEE, pp.1-8, 2015.

[39] Tejas, R. and H, M.D.T, "Design and implementation of an efficient routing schemes to increase distribute lifetime efficiency in WSN", IEEE, pp. 237-240, 2016

[40] Thein, M.Ch.M. and Thein, Th., "An energy efficient cluster-head selection for wireless sensor networks", IEEE International Conference on Intelligent Systems, Modelling and Simulation, pp. 287-291, 2010.

[41] Wang, J., Yin, Y., Kim, J. and Lee, S., "A mobile sink based energy efficient clustering algorithm for WSNs", IEEE, pp.678-683, 2012.

[42] Wu, M., Liu, H. and Min, Q., "Lifetime Enhancement by Cluster Head Evolutionary Energy Efficient Routing Model for WSN," IEEE, pp. 545-548, 2016.

[43] Xiuwu, Y., Lixing, F.F.Z. and Feng,Z., "WSN Monitoring Area Partition Clustering Routing Algorithm for EnergyBalanced," IEEE, pp. 80-84, 2016.

[44] Yadav, L. and Sunitha, C., "Low Energy Adaptive Clustering Hierarchy in WSN (LEACH) ", International Journal of Computer Science, Networking, vol.5, pp. 4661-4664, 2014.

[45] Zhang, Y. and Yuan, X., "Fault Diagnosis in Clustering WSN Based Neighbor Cooperation", IEEE, pp.1803-1807, 2016.

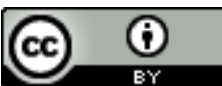

This work is licensed under a Creative Commons Attribution 4.0 International License.

DOI:10.24297/ijct.v16i5.6264 